\title{
A THEOREM ON ANALYTIC MAPPINGS OF COMPLEX MANIFOLDS
}

\author{
MINORU KURITA
}

Dedicated to late professor TADASI NAKAyama

We prove in this paper a theorem on analytic mappings of the complex space $C_{n}$ into the complex projective space $P_{n}$. The theorem is closely related to that of S. S. Chern in [1], and the main idea of the proof is the same with the latter, though the calculations are rather different. The background of our calculation is the normal contact metric structure which was found by $\mathrm{S}$. Sasaki and Y. Hatakeyama [4].

Our purpose is to find a criterion for an analytic mapping $f$ of $C_{n}$ into $P_{n}$ in order that $f\left(C_{n}\right)$ covers almost every part of $P_{n}$. We take cartesian coordinates $z^{1}, \ldots, z^{n}$ in $C_{n}$ and then the metric is given by

$$
d \Sigma^{2}=\sum_{j=1}^{n} d z^{j} d \bar{z}^{j}
$$

As for the elliptic metric of $P_{n}$ we have

$$
d T^{2}=\left(1+|w|^{2}\right)^{-2}\left(\sum_{j=1}^{n} d w^{j} d \bar{w}^{j}+\sum_{j<k}\left|d w^{j} w^{k}-d w^{k} w^{j}\right|^{2}\right)
$$

in complex coordinates $w^{1}, \ldots, w^{n}$, where we have put $|w|=\left(\sum w^{j} \bar{w}^{j}\right)^{1 / 2}$. An analytic mapping $f: C_{n} \rightarrow P_{n}$ can be represented by

$$
w^{j}=f_{j}\left(z^{1}, \ldots, z^{n}\right) \quad(j=1, \ldots, n),
$$

where $f_{j}\left(z^{1}, \ldots, z^{n}\right)$ are analytic functions. We put

$$
f^{*}\left(d T^{2}\right)=\sum_{j k} a_{j k} d z^{j} d \bar{z}^{k}
$$

( $\left.a_{j k}\right)$ is a hermitian tensor on $C_{n}$ which is determined by the mapping $f$. We denote the eigenvalues of $\left(a_{j k}\right)$ by $\lambda_{1}, \ldots, \lambda_{n}$ and put

$$
B=\sum_{j=1}^{n} \lambda_{1} \lambda_{2} \cdots \lambda_{j-1} \lambda_{j+1} \cdots \lambda_{n} .
$$

Received June 23, 1965. 
We use the notations

$D_{r}:$ the sphere $|z| \leqq r$ in $C_{n}, \quad S_{r}=\partial D_{r}, \quad D\left(r_{0}, r\right)=D_{r}-D_{r_{0}}$

$\Omega$ : the volume element in $P_{n}$ determined by the metric $d T^{2}$.

We define $v\left(D_{r}\right)$ and $T_{k}(r)$ by

$$
v\left(D_{r}\right)=\int_{D_{r}} f^{*} \Omega, \quad T_{k}(r)=\int_{r_{0}}^{r} \frac{v\left(D_{r}\right)}{r^{k}} d r
$$

where $r_{0}$ is a constant smaller than $r$. We denote by $\Theta$ an element of the solid angle about the origin of $C_{n}$ and then the volume element in $C_{n}$ can be given by

$$
\Pi=r^{2 n-1} d r \wedge \Theta
$$

where $|z|=r$. We put moreover

$$
U(r)=\int_{D\left(r_{0}, r\right)} \frac{B}{r^{2 n}} \Pi, \quad Y(r)=\frac{1}{I_{2 n-1}} \int_{S_{r}} B r^{2 n-2} \Theta,
$$

where $I_{2 n-1}$ is the surface area of a unit sphere in $C_{n}$.

Now S. S. Chern's theorem in [1] is in our version

TheOREM A. We assume that for $r \rightarrow \infty$ we have

$$
T_{2 n-1}(r) \rightarrow \infty \quad \text { and } \quad U(r)=O\left(T_{2 n-1}(r)\right) .
$$

Then the complement of $f\left(C_{n}\right)$ is of measure zero.

On the other hand our theorem is

Theовем B. We denote the measure of the complement of $f\left(D_{r}\right)$ by $b$, and the total measure of $P_{n}$ by $c\left(=\pi^{n} / n !\right)$. Then we have an inequality

$$
\frac{b}{c} \leqq a \frac{Y(r)}{T_{1}(r)}
$$

where $a=\frac{1}{4}\left(1+\frac{1}{2}+\cdots+\frac{1}{n}\right)$.

Hence if

$$
\lim _{r \rightarrow \infty} \frac{Y(r)}{T_{1}(r)}=0
$$

the complement of $f\left(C_{n}\right)$ is of measure zero.

Theorem A lacks an example and even for the most simple mapping

$$
w^{j}=z^{j} \quad(j=1,2, \ldots, n)
$$


the assumptions do not hold good, because $T_{2 n-1}(r)$ and $U(r)$ are finite for $r \rightarrow \infty$ as we shall show afterwards. On the other hand we have

$$
T_{1}(r)=O(\log r), \quad Y(r) \rightarrow 1
$$

for $r \rightarrow \infty$ and (0.9) is an example of theorem B. We will prove theorem B and also give formulas to calculate $T_{k}(r), Y(r)$ and $U(r)$. Hereafter we omit the summation symbol $\sum$ for double indices and we assume throughout that the indices run as follows

$$
j, k=1,2, \ldots, n \quad a, b=2,3, \ldots, n .
$$

\section{Decomposition of Kaehlerian metrics of $C_{n}$ and $P_{n}$}

A Kaehlerian metric on an $n$-dimensional complex analytic manifold $M$ is a real analytic positive definite Riemannian metric which can locally be represented as

$$
d \Sigma^{2}=g_{j k}(z, \bar{z}) d z^{j} d \bar{z}^{k} \quad\left(g_{j k}=\bar{g}_{k j}\right)
$$

satisfying the relation $d\left(g_{j k} d z^{j} \wedge d \bar{z}^{k}\right)=0$. When we take suitable local coframes

$$
\sigma^{j}=p_{k}^{j}(z, \bar{z}) d z^{k} \quad(j=1,2, \ldots, n),
$$

we have

$$
d \Sigma^{2}=\sigma^{j} \bar{\sigma}^{j}, \quad d\left(\sigma^{j} \wedge \bar{\sigma}^{j}\right)=0 .
$$

Kaehlerian metric of $C_{n}$

This is given by

$$
d \Sigma^{2}=(d z, d z)=d z^{j} d \bar{z}^{j} .
$$

We put

$$
r=|z|=(z, z)^{1 / 2}, \quad r^{-1} z=\left(u^{1}, \ldots, u^{n}\right)
$$

and we get

$$
d \Sigma^{2}=d r^{2}+r^{2}(d u, d u) .
$$

We take vectors $u_{a}=\left(u_{a}^{1}, u_{a}^{2}, \ldots, u_{a}^{n}\right)(a=2,3, \ldots, n)$ which constitute a unitary base together with $u=\left(u^{1}, \ldots, u^{n}\right)$ and put

$$
\theta=-i(d u, u), \quad \mu^{a}=\left(d u, u_{a}\right) .
$$

$\theta$ is a real form and we have

$$
d \theta=i \mu^{a} \wedge \bar{\mu}^{a} .
$$

The metric reduces to 


$$
(d u, d u)=\theta^{2}+\mu^{a} \bar{\mu}^{a}
$$

on a unit sphere $|u|=1$. We have by (1.2) (1.5)

$$
d \Sigma^{2}=d r^{2}+r^{2} \theta^{2}+r^{2} \mu^{a} \bar{\mu}^{a} .
$$

When we put

$$
\mu^{a}=\theta^{a}+i \theta^{a+n} \quad\left(\theta^{a}, \theta^{a+n} \text { are real forms }\right),
$$

we get

$$
d \Sigma^{2}=d r^{2}+r^{2} \theta^{2}+r^{2}\left(\theta^{a} \theta^{a}+\theta^{a+n} \theta^{a+n}\right)
$$

and the volume element of $C_{n}$ is given by

$$
\Pi=d r \wedge r \theta \wedge r \theta^{2} \wedge \cdots \wedge r \theta^{n} \wedge r \theta^{n+2} \wedge \cdots \wedge r \theta^{2 n} .
$$

We take an orientation of $C_{n}$ in such a way that this form is positive. We put

$$
\Theta_{0}=\theta^{2} \wedge \cdots \wedge \theta^{n} \wedge \theta^{n+2} \wedge \cdots \wedge \theta^{2 n}, \quad \Theta=\theta \wedge \Theta_{0}
$$

and we get

$$
\Pi=r^{2 n-1} d r \wedge \Theta
$$

The element of the area on the sphere $r=$ const. is $r^{2 n-1} \Theta$ and hence $\Theta$ is an element of a solid angle about the origin. Next we put

$$
\sigma^{1}=d r+i r \theta, \quad \sigma^{a}=r \mu^{a} .
$$

Then we have

$$
\sigma^{1}=r^{-1}(d z, z), \quad \sigma^{a}=\left(d z, u_{a}\right)
$$

and these do not contain $d \bar{z}^{1}, \ldots, d \bar{z}^{n}$. Thus

$$
d \Sigma^{2}=\sigma^{1} \bar{\sigma}^{1}+\sigma^{a} \bar{\sigma}^{a}=\sigma^{j} \bar{\sigma}^{j},
$$

where the sum with respect to $j$ ranges from 1 to $n$. When we represent $\Theta_{0}$ by $\mu^{a}$ we get

$$
\Theta_{0}=(-1)^{n(n-1) / 2}(2 i)^{-n+1} \mu^{2} \wedge \cdots \wedge \mu^{n} \wedge \bar{\mu}^{2} \wedge \cdots \wedge \bar{\mu}^{n} .
$$

Kaehlerian metric of $P_{n}$

We take a unit vector $p=\left(p^{0}, p^{1}, \ldots, p^{n}\right)$ in $C_{n+1}$. We construct the $n$-dimensional projective complex space $P_{n}$ in a usual way. The elliptic metric on $P_{n}$ is given by 


$$
d T^{2}=(d p, d p)-(d p, p)(p, d p)
$$

and when we put

$$
w^{j}=p^{j} / p^{0} \quad(j=1,2, \ldots, n),
$$

we get (0.2). We take a unitary base $e_{0}, e_{1}, \ldots, e_{n}$ in $C_{n+1}$ and for any unit vector $p$ in $C_{n+1}$ we define $t$ by $\cos t=\left(e_{0}, p\right)\left(0 \leqq t \leqq \frac{\pi}{2}\right)$ and decompose $p$ as $p=a e_{0}+b v_{1}$ where $\left(e_{0}, v_{1}\right)=0$ and $\left|v_{1}\right|=1$. Then $|a|=\cos t,|a|^{2}+|b|^{2}=1$. We take suitable real numbers $\alpha, \beta$ and put $e^{i \beta} v_{1}=v$. Then we get $e^{i \alpha} p=\cos t \cdot e_{0}+$ $\sin t \cdot v$, where $\left(e_{0}, v\right)=0,|v|=1$. When we use $e^{i \alpha} p$ instead of $p$ in (1.15), $d T^{2}$ is the same. We put

$$
p=\cos t \cdot e_{0}+\sin t \cdot v
$$

into (1.15) and we get

$$
d T^{2}=d t^{2}+\sin ^{2} t \cos ^{2} t \cdot \varphi^{2}+\sin ^{2} t \cdot \nu^{a} \bar{\nu}^{a},
$$

where we have put

$$
\varphi=-i(d v, v), \quad \nu^{a}=\left(d v, v_{a}\right),
$$

$v_{a}(a=2,3, \ldots, n)$ being vectors which constitute a unitary base together with $v$ in the complex hyperplane orthogonal to $e_{0}$. We put moreover

$$
\nu^{a}=\varphi^{a}+i \varphi^{a+n} \quad\left(\varphi^{a}, \varphi^{a+n} \text { are real forms }\right)
$$

and then

$$
d T^{2}=d t^{2}+\sin ^{2} t \cos ^{2} t \cdot \varphi^{2}+\sin ^{2} t\left(\varphi^{a} \varphi^{a}+\varphi^{a+n} \varphi^{a+n}\right)
$$

and the volume element of $P_{n}$ is

$$
\Omega=d t \wedge \sin t \cos t \varphi \wedge \sin t \cdot \varphi^{2} \wedge \cdots \wedge \sin t \cdot \varphi^{n} \wedge \sin t \cdot \varphi^{n+2} \wedge \cdots \wedge \sin t \cdot \varphi^{2 n} .
$$

We take an orientation of $P_{n}$ in such a way that this form is positive definite. We put

$$
\Phi_{0}=\varphi^{2} \wedge \cdots \wedge \varphi^{n} \wedge \varphi^{n+2} \wedge \cdots \wedge \varphi^{2 n}, \quad \Phi=\varphi \wedge \Phi_{0},
$$

then we get

$$
\Omega=\sin ^{2 n-1} t \cos t d t \wedge \emptyset .
$$

The element of area on a sphere $t=$ const. is $\sin ^{2 n-1} t \cos t \cdot \Phi$. Hence $\Phi$, which is the limit of $\sin ^{2 n-1} t \cos t \cdot \Phi / t^{2 n-1}$ for $t \rightarrow 0$, is an element of a solid angle about the origin. We have 


$$
d \Phi=0 .
$$

We have by virtue of (1.16) (1.17) and $|p|=1$

$$
\cos t=p^{0}=\left(1+|w|^{2}\right)^{-1 / 2} .
$$

As $\sin t \cdot v=\left(0, p^{1}, \ldots, p^{n}\right)=\left(0, p^{0} w^{1}, \ldots, p^{0} w^{n}\right)$, we have

$$
v=\left(0, w^{1} /|w|, \ldots, w^{n} /|w|\right) \text {. }
$$

We put

$$
\tau^{0}=d t+i \sin t \cos t \cdot \varphi, \quad \tau^{a}=\sin t \cdot \nu^{a} .
$$

We get by (1.19) (1.25) (1.18)

$$
\begin{gathered}
\tau^{1}=(d w, v)\left(1+|w|^{2}\right)^{-1}, \quad \tau^{a}=\left(d w, v_{a}\right)\left(1+|w|^{2}\right)^{-1 / 2}, \\
d T^{2}=\tau^{1} \bar{\tau}^{1}+\tau^{a} \bar{\tau}^{a}=\tau^{j} \bar{\tau}^{j} .
\end{gathered}
$$

When we represent $\Phi_{0}$ by $\nu^{a}$, we get

$$
\Phi_{0}=(-1)^{n(n+1) / 2}(2 i)^{-n+1} \nu^{2} \wedge \cdots \wedge \nu^{n} \wedge \bar{\nu}^{2} \wedge \cdots \wedge \bar{\nu}^{n} .
$$

As $\nu^{a} \bar{\nu}^{a}$ is an elliptic metric on $P_{n-1}$ (cf. [3] p. 314), we have

$$
d \Phi_{0}=0 .
$$

\section{The first main theorem}

The following formulation of the first main theorem of differentiable mapping is due to S. S. Chern. (cf. [2]) Let $M$ be an $m$-dimensional dfferentiable manifold and $N$ be a compact orientable Riemannian manifold of the same dimension. We consider a differentiable mapping $M \rightarrow N$. We denote the surface area of an $m$-dimensional unit sphere by $I_{m-1}$, the volume element of $N$ by $\Omega$ and the total measure of $N$ by $c$. We take a point $a$ in $N$. Then there exists a function $u=u(x)$ on $N$ satisfying the following conditions.

$$
-\frac{1}{I_{m-1}} d(* d u)=\frac{1}{c} \Omega
$$

(ii) on a certain neighborhood of the point $a$ we have

$$
* d u=(1+\varepsilon) \Phi_{a},
$$

where $\Phi_{a}$ is an element of a solid angle at $a$ and $\varepsilon \rightarrow 0$ as $x$ tends to $a$.

Let $f$ be a differentiable mapping $M \rightarrow N$ and $D$ be a domain on $M$. We assume that for a point $a$ of $f(M)$ the set $f^{-1}(a) \cap D$ consists of a finite number 
of points $p_{1}, \ldots, p_{k}$, which are interior points of $D$, and $f^{-1}(a) \cap \partial D$ is empty. We denote by $n(a)$, or more precisely $n(a, D)$, the degree of the mapping $f$ about the point $a$. This is the sum of the degree of the mappings of small neighborhoods of each points $p_{1}, \ldots, p_{k}$. We take functions above stated and put

$$
\lambda=-\frac{1}{I_{m-1}} f^{*}(* d u)
$$

Then $n(a)$ can be represented as

$$
n(a)=\frac{1}{c} \int_{D} f^{*} \Omega-\int_{\partial D} \lambda
$$

This is the first main theorem. The proof can be given by applying the Stokes' theorem to the form $\lambda$ and the domain $D-\sum_{j=1}^{k} D_{j}(\varepsilon)$ and making $\varepsilon$ tend to 0 , where $D_{j}(\varepsilon)$ is a sphere of radius $\varepsilon$ with respect to local coordinates about the point $p_{j}$.

\section{The function $u$ on $P_{n}$.}

The function $u$ which satisfies (i) and (ii) is determined uniquely up to an additive constant. We will find the one for $P_{n}$. We take the point $a$ at $t=0$ and put $u=u(t)$, we have $d u=u^{\prime} d t \quad\left(u^{\prime}=d u / d t\right)$ and by (1.21)

$$
\begin{aligned}
& * d u=u^{\prime}(\sin t \cos t \cdot \varphi) \wedge \sin ^{2 n-2} t \cdot \varphi^{2} \wedge \cdots \wedge \varphi^{n} \wedge \varphi^{n+2} \wedge \cdots \wedge \varphi^{2 n} \\
& \quad=u^{\prime} \sin ^{2 n-1} t \cos t \cdot \emptyset \\
& d(* d u)=\left(u^{\prime} \sin ^{2 n-1} t \cos t\right)^{\prime} d t \wedge \emptyset .
\end{aligned}
$$

As $\Omega=\sin ^{2 n-1} t \cos t \cdot d t \wedge \Phi$ by (1.23) in our case, we get

Hence by (ii)

$$
\left(u^{\prime} \sin ^{2 n-1} t \cos t\right)^{\prime}=-2 n \sin ^{2 n-1} t \cos t .
$$

$$
\begin{gathered}
u^{\prime} \sin ^{2 n-1} t \cos t=1-\sin ^{2 n} t \\
* d u=\left(1-\sin ^{2 n} t\right) \Phi
\end{gathered}
$$

and also

$$
u=\log \sin t-\sum_{k=1}^{n-1} \frac{1}{2 k \sin ^{2 k} t}
$$

\section{Integrated form of the first main therem}

We take a sphere $D_{r}:|z| \leqq r$ in $C_{n}$ and put $\partial D_{r}=S_{r}$, and apply the first main theorem to the mapping $f: C_{n} \rightarrow P_{n}$. Then we have by (2.2) 


$$
n\left(a, D_{r}\right)=\frac{1}{c} \int_{D r} f^{*} \Omega-\int_{S_{r}} \lambda .
$$

We divide by $r^{k}$ ( $k$ const. ) and integrate from $r_{0}$ to $r$ with respect to $r$. Putting

$$
N_{k}\left(a, D_{r}\right)=\int_{r_{0}}^{r} \frac{n\left(a, D_{r}\right)}{r^{k}} d r
$$

and taking (0.6) into consideration we get

$$
N\left(a, D_{r}\right)=\frac{1}{c} T_{k}(r)+\frac{1}{I_{2 n-1}} J_{k}
$$

where

$$
J_{k}=-I_{2 n-1} \int_{D\left(r_{0}, r\right)} \Lambda
$$

with

$$
\begin{aligned}
\Lambda & =-I_{2 n-1} r^{-k} d r \wedge \lambda=r^{-k} d r \wedge f^{*}(* d u) \\
& =r^{-k} d r \wedge f^{*}\left(\left(1-\sin ^{2 n} t\right) \emptyset\right) .
\end{aligned}
$$

Estimation of $J_{k}$

We take coframes in $C_{n}$ and $P_{n}$ as in (1.13) and (1.28). We can put

$$
f^{*} \tau^{j}=p_{k}^{j} \sigma^{k}
$$

by virtue of (0.3) (1.12) (1.27). Hence

$$
f^{*}\left(d \Sigma^{2}\right)=a_{j k} \sigma^{j} \bar{\sigma}^{k} \quad\left(a_{j k}=p_{j}^{h} \bar{p}_{k}^{h}\right) .
$$

Hereafter we often omit conventionally such notation $f^{*}$ as in (3.4) (3.5) (3.6). We consider such a case that $\operatorname{det}\left(a_{j k}\right) \neq 0$, namely $\operatorname{det}\left(p_{j}^{k}\right) \neq 0$. Then we can put by (3.5)

$$
\begin{gathered}
\sigma^{1}=q_{1}^{1} \tau^{1}+q_{a}^{1} \tau^{a}, \quad \sigma^{a}=q_{1}^{a} \tau^{1}+q_{b}^{a} \tau^{b} . \\
(a, b=2, \ldots, n)
\end{gathered}
$$

We have by (1.11) (1.26)

$$
\begin{gathered}
d r+i r \theta \equiv q_{1}^{1}(d t+i \sin t \cos t \cdot \varphi) \\
d r-i r \theta \equiv \bar{q}_{1}^{1}(d t-i \sin t \cos t \cdot \varphi) . \\
\left(\bmod \nu^{2}, \nu^{3}, \ldots, \nu^{n}\right)
\end{gathered}
$$

Hence we get

$$
\sin t \cos t d r \wedge \varphi \wedge \Phi_{0}=r d t \wedge \theta \wedge \Phi_{0} .
$$

This can be verified by solving (3.8) with respect to $d r, r \theta$ and putting $d r$ 
into the right side and $r \theta$ into the left side. Here we assumed $\operatorname{det}\left(p_{j}^{k}\right) \neq 0$. But (3.9) is an algebraic consequence of (3.5) and so (3.9) is true for the case $\operatorname{det}\left(p_{j}^{k}\right)=0$, too.

As $\mathscr{D}=\varphi \wedge \mathscr{D}_{0}$, we get by (3.4) (3.9)

$$
\Lambda=\frac{1-\sin ^{2 n} t}{\sin t \cos t} r^{-k+1} d t \wedge \theta \wedge \Phi_{0} .
$$

We put

$$
v=-\log \sin t+\frac{1}{2} \sum_{k=1}^{n-1}(-1)^{k-1} \frac{1}{k}\left(\begin{array}{c}
n-1 \\
k
\end{array}\right) \cos ^{2 k} t .
$$

The second term on the right side is one obtained by putting $s=\cos ^{2} t$ in the integral $\int_{1}^{s} \frac{1-(1-s)^{n-1}}{s} d s$. Hence

$$
v \geqq 0 \quad \text { for } \quad \frac{\pi}{2} \geqq t>0 .
$$

We get by (3.11)

$$
v^{\prime}=\frac{d v}{d t}=-\frac{1-\sin ^{2 n} t}{\sin t \cos t}
$$

Hence by the relation $d \Phi_{0}=0$

$$
\Lambda=-r^{k+1} d v \wedge \theta \wedge \Phi_{0}=d\left(-r^{k+1} v \theta \wedge \Phi_{0}\right)+v d\left(r^{-k+1} \theta\right) \wedge \Phi_{0} .
$$

Hence we have

$$
J_{k}=\int_{D\left(r_{0}, r\right)} A=-\int_{s_{r}} r^{-k+1} v \theta \Phi_{0}+\int_{S_{r_{0}}} r^{-k+1} v \theta \Phi_{0}+\int_{D\left(r_{0}, r\right)} v d\left(r^{-k+1} \theta\right) \cdot \Phi_{0}
$$

By (1.11) (1.26) (3.5)

$$
\begin{gathered}
\sin t \cdot \nu^{a} \equiv p_{b}^{a} r \mu^{b} . \quad\left(\bmod \sigma^{1}=d r+i r \theta\right) \\
(a, b=2, \ldots, n)
\end{gathered}
$$

We put

$$
\Delta=\operatorname{det}\left(p_{b}^{a}\right)
$$

and we get by (1.9) (1.28)

$$
\sin ^{2 n-2} t \cdot \Phi_{0} \equiv r^{2 n-2} \Delta \bar{\Delta} \Theta_{0} . \quad(\bmod d r, \theta)
$$

As we have $d r=0$ on $S_{r}$, we get

$$
\sin ^{2 n-2} t \cdot \theta \wedge \Phi_{0}=r^{2 n-2} \Delta \bar{\Delta} \theta \wedge \Theta_{0} .
$$

Hẹce taking $\Theta=\theta \wedge \Theta_{0}$ into consideration 


$$
r^{-k+1} v \theta \wedge \Phi_{0}=v \sin ^{-2 n+2} t \cdot \Delta \bar{\Delta} r^{2 n-k-1} \Theta .
$$

By (1.4) and (1.11) we have

$$
d \theta=i \mu^{a} \wedge \bar{\mu}^{a}=i r^{-2} \sigma^{a} \wedge \bar{\sigma}^{a}, \quad \sigma^{1} \wedge \bar{\sigma}^{-1}=-2 i r d r \wedge \theta .
$$

Hence we get

$$
\begin{aligned}
d\left(r^{-k+1} \theta\right) \wedge \Phi_{0} & =r^{-k+1} d \theta \wedge \Phi_{0}-(k-1) r^{-k} d r \wedge \theta \wedge \Phi_{0} \\
& =i r^{-k-1}\left(\sigma^{1} \wedge \bar{\sigma}^{1}+\sigma^{a} \wedge \bar{\sigma}^{a}\right) \wedge \Phi_{0}-(k+1) r^{-k} d r \wedge \theta \wedge \Phi_{0} \\
& =i r^{-k-1}\left(\sigma^{j} \wedge \bar{\sigma}^{j}\right) \wedge \Phi_{0}-\left(k+1 \mid r^{-k} d r \wedge \theta \wedge \Phi_{0} .\right.
\end{aligned}
$$

By virtue of (3.16)

$$
d r \wedge \theta \wedge \Phi_{0}=r^{2 n-2} \sin ^{-2 n+2} t \cdot \bar{\Delta} d r \wedge \theta \wedge \Theta_{0}
$$

and also

$$
i\left(\sigma^{j} \wedge \bar{\sigma}^{j}\right) \wedge \Phi_{0}=2 C_{0} r^{2 n-3} \sin ^{-2 n+2} t d r \wedge \theta \wedge \Theta_{0} .
$$

Here

$$
C_{0}=\operatorname{det}\left(c_{a b}\right), \quad c_{a b}=p_{j}^{a} \bar{p}_{j}^{b} . \quad(a, b=2, \ldots, n)
$$

(3.21) can be verified as follows. By (1.29) and (1.26)

$$
\sin ^{2 n-2} t \cdot \Phi_{0}=(-1)^{n(n-1) / 2}(2 i)^{-n+1} \tau^{2} \wedge \cdots \wedge \tau^{n} \wedge \bar{\tau}^{2} \wedge \cdots \wedge \bar{\tau}^{n} .
$$

We put $\tau^{a}=p_{j}^{a} \sigma^{j}$ into the right side and multiply by $\sigma^{j} \wedge \bar{\sigma}^{j}$. When we put

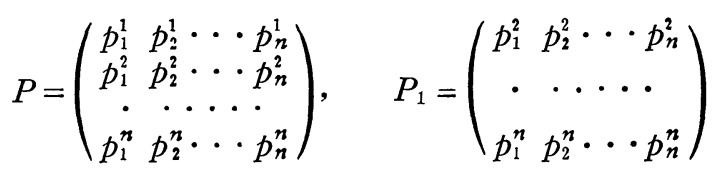

and denote by $\Delta_{j}$ the minor determinant corresponding to $p_{j}^{1}$ in $P$, we get

$$
\begin{aligned}
& \sigma^{j} \wedge \bar{\sigma}^{j} \wedge \tau^{2} \wedge \cdots \wedge \tau^{n} \wedge \bar{\tau}^{2} \wedge \cdots \wedge \bar{\tau}^{n} \\
= & \Delta_{j} \bar{\Delta}_{j} \sigma^{1} \wedge \bar{\sigma}^{1} \wedge \sigma^{2} \wedge \sigma^{3} \wedge \cdots \wedge \sigma^{n} \wedge \bar{\sigma}^{2} \wedge \bar{\sigma}^{3} \wedge \cdots \wedge \bar{\sigma}^{n} \\
= & \operatorname{det}\left(P_{1}^{t} \bar{P}_{1}\right)(-2 i r) d r \wedge \theta \wedge \sigma^{2} \wedge \cdots \wedge \sigma^{n} \wedge \bar{\sigma}^{2} \wedge \cdots \wedge \bar{\sigma}^{n} .
\end{aligned}
$$

As $C_{0}=\operatorname{det}\left(P_{1}^{t} \bar{P}_{1}\right)$, we get the verification of (3.21) by virtue of (1.11) and (1.14).

By putting (3.20) (3.21) into (3.19) and taking $\Theta=\theta \wedge \Theta_{0}$ into consideration we get

$$
d\left(r^{-k+1} \theta\right) \wedge \emptyset_{0}=2 r^{2 n-k-1} \sin ^{-2 n+2} t\left(C_{0}-\frac{k+1}{2} \Delta \bar{\Delta}\right) d r \wedge \Theta
$$


By (3.18) (3.24) we get from (3.14)

$$
\begin{array}{rl}
J_{k}=-\int_{S_{r}} v \sin ^{-2 n+2} & t \cdot \Delta \bar{\Delta} r^{2 n-k-1} \Theta+\int_{S_{r_{0}}} v \sin ^{-2 n+2} t \cdot \Delta \bar{\Delta} r^{2 n-k-1} \Theta \\
& +\int_{\left.D^{(} r_{0}, r\right)} 2 v r^{2 n-k-1} \sin ^{-2 n+2} t\left(C_{0}-\frac{k+1}{2} \Delta \bar{\Delta}\right) d r \Theta .
\end{array}
$$

$C_{0}$ determined by $(3.22)$ is a minor determinant corresponding to $c_{11}$ of the matrix $P^{t} \bar{P}=\left(c_{j k}\right)(j, k=1,2, \ldots, n)$ and $P^{t} \bar{P}$ is a hermitian matrix which is positive definite or semi-definite. Hence $C_{0}$ is not greater than the sum of principal determinants of $n-1$ - th order of $P^{t} \bar{P}$, namely the sum of products of $n-1$ numbers taken arbitrarily from $n$ eigenvalues of $P^{t} \bar{P}$. On the other hand we have by (3.6)

$$
\left(a_{j k}\right)={ }^{t} P \bar{P}=\bar{P}^{-1} \overline{\left(P^{t} \bar{P}\right)} \bar{P}
$$

and so the eigenvalues of $\left(a_{j k}\right)$ are the same with those of $\overline{P^{t} \overline{\bar{P}}}$ namely of $P^{t} \bar{P}$. Hence by the definition of $B$ in $(0.5)$ we have

$$
C_{0} \leqq B \text {. }
$$

By taking (3.15) (3.23) into consideration we have

$$
\Delta \bar{\Delta} \leqq C_{0} \leqq B .
$$

Hence by (3.12) (3.25) (3.27) we get for $k=1$

$$
J_{1} \geqq-\int_{S_{r}} v \sin ^{-2 n+2} t B r^{2 n-2} \Theta .
$$

This is the most important key to our proof of theorem B.

\section{The final step of the proof}

We denote the complement of $f\left(D_{r}\right)$ by $K$, whose measure $b$ we will estimate. For any point $a$ in $K$ we have $n\left(a, D_{r}\right)=0$ and so by (3.2) we get

$$
\frac{1}{c} T_{1}(r)+\frac{1}{I_{2 n-1}} J_{1}=0
$$

Hence we get by (3.28)

$$
\frac{1}{c} I_{2 n-1} T_{1}(r) \leqq \int_{S_{r}} v \sin ^{-2 n+2} t \cdot B r^{2 n-2} \Theta
$$

We multiply the both sides by the invariant volume element $\Omega$ of $P_{n}$ with 
respect to the point $a$ and integrate on $K$. Then we have

$$
\frac{b}{c} I_{2 n-1} T_{1}(r) \leqq \int_{S_{r}}\left(\int_{K} v \sin ^{-2 n+2} t \cdot \Omega\right) B r^{2 n-2} \Phi .
$$

In the first we fix a point $p$ on $S_{r}$ and estimate the integral in the bracket on the right side of (4.3). As $t$ is a distance from the point $a$ to the point $p$ and $\Omega$ is the invariant measure, we get by $(1.23)$

$$
\Omega=\sin ^{2 n-1} t \cos t d t \wedge \Phi,
$$

when we take coordinates such that $t=0$ for the point $p$. Hence we get

$$
\int_{K} v \sin ^{-2 n+2} t \cdot \Omega \leqq \int_{P_{n}} v \sin t \cos t d t \cdot \Phi
$$

Here we have $\int \Phi=I_{2 n-1}$ and by (3.13)

$$
\begin{aligned}
\int_{0}^{\pi / 2} v & \sin t \cos t d t=\left[v \frac{1}{2} \sin ^{2} t\right]_{0}^{\pi / 2}-\int_{0}^{\pi / 2} \frac{d v}{d t} \frac{1}{2} \sin ^{2} t d t \\
& =\frac{1}{2} \int_{0}^{\pi / 2}\left(1-\sin ^{2 n} t\right) \frac{\sin t}{\cos t} d t=\frac{1}{4} \int_{0}^{1} \frac{1-s^{n}}{1-s} d s \quad\left(s=\sin ^{2} t\right) \\
& =\frac{1}{4}\left(1+\frac{1}{2}+\cdots+\frac{1}{n}\right)=a .
\end{aligned}
$$

Thus by (4.3)

$$
\frac{b}{c} I_{2 n-1} T_{1}(r) \leqq a \int_{s_{r}} B r^{2 n-2} \emptyset=a I_{2 n-1} Y(r),
$$

namely

$$
\frac{b}{c} \leqq a \frac{Y(r)}{T_{1}(r)}
$$

This is theorem $B$.

5. Explicit calculations of $T_{1}(r), T_{2 n-1}(r), Y(r)$ and $U(r)$

In the last we will show how to calculate these numbers for the mapping (0.3) and will actually calculate in the case (0.9). In this section we do not omit the symbol $\sum$ representing the summation.

In the first we denote by $P_{j}$ a matrix obtained from $P$ by omitting the $j$-th row and by $\Delta_{k}^{j}$ the minor determinant of $P$ corresponding to $p_{k}^{j}$. Then we have

$$
B=\sum_{j} \operatorname{det}\left(P_{j}^{t} \bar{P}_{j}\right)=\sum_{j_{0} \kappa} \Delta_{k}^{j} \bar{\Delta}_{k}^{j},
$$


Now we put

$$
T^{j}=\tau^{1} \wedge \tau^{2} \wedge \cdots \wedge \tau^{j-1} \wedge \tau^{j+1} \wedge \cdots \wedge \tau^{n}
$$

We have by (3.5) (0.5)

$$
\left(\sum_{j} \sigma^{j} \wedge \bar{\sigma}^{j}\right) \wedge\left(\sum_{k} T^{k} \wedge \bar{T}^{k}\right)=(-1)^{n-1} B \sigma^{1} \wedge \cdots \wedge \sigma^{n} \wedge \bar{\sigma}^{1} \wedge \cdots \wedge \bar{\sigma}^{n}
$$

Taking (1.26) into consideration we put

$$
\sigma^{j}=d z^{j}, \quad \tau^{1}=\left(d w, v\left(1+|w|^{2}\right)^{-1}, \quad \tau^{a}=\left(d w, v_{a}\right)\left(1+|w|^{2}\right)^{-1 / 2} .\right.
$$

As $v_{a}(a=2, \ldots, n)$ constitute a unitary base with $v=w|w|^{-1}$ we get after some calculation

$$
\begin{aligned}
& T^{1}=\tau^{2} \wedge \cdots \wedge \tau^{n} \\
& \left.=\varepsilon\left(1+|w|^{2}\right)^{-(n-1) / 2}|w|^{-1} \sum_{j}-1\right)^{j-1} \bar{w}^{j} d w^{1} \wedge \cdots \wedge \widehat{d w^{j}} \wedge \cdots \wedge d w^{n} \\
& T^{a}=\tau^{1} \wedge \cdots \wedge \hat{\tau}^{a} \wedge \cdots \wedge \tau^{n} \\
& =(-1)^{a} \varepsilon\left(1+|w|^{2}\right)^{-n / 2} \sum_{\jmath}(-1)^{j-1} v_{a}^{j} d w^{1} \wedge \cdots \wedge \widehat{d w}^{j} \wedge \cdots \wedge d w^{n},
\end{aligned}
$$

where $\wedge$ means that the indicated term is absent in the product, and $\varepsilon$ is a determinant formed by the components of $v, v_{2}, \ldots, v_{n}$. We put these into the left side of (5.1) and get

$$
B=\left(1+|w|^{2}\right)^{-n}\left(\sum_{j} K_{j j}+\sum_{i, j} \bar{w}^{i} w^{j} K_{i j}\right),
$$

where $K_{i j}$ is a cofactor corresponding to $k_{i j}$ of the matrix $K=\left(k_{i j}\right)$ defined by

$$
J=\left(\begin{array}{c}
\frac{\partial w^{1}}{\partial z^{1}} \cdots \frac{\partial w^{1}}{\partial z^{n}} \\
\cdots \cdots \\
\frac{\partial w^{n}}{\partial z^{1}} \cdots \frac{\partial w^{n}}{\partial z^{n}}
\end{array}\right) . \quad K=J^{t} \bar{J} .
$$

On the other hand we have

$$
\tau^{1} \wedge \tau^{2} \wedge \cdots \wedge \tau^{n}=\left(1+|w|^{2}\right)^{-(n+1) / 2} \varepsilon d w^{1} \wedge d w^{2} \wedge \cdots \wedge d w^{n} .
$$

and the volume element $\Omega$ of $P_{n}$ is given by

$$
\Omega=\left(1+|w|^{2}\right)^{-(n+1)} \operatorname{det} K \cdot r^{2 n-1} d r \wedge \Theta .
$$

For the special case $w^{j}=f_{j}\left(z^{j}\right) \quad(j=1,2, \ldots, n)$ we have

$$
B=\left(1+|w|^{2}\right)^{-n} \sum_{j}\left(1+\left|f_{j}\right|^{2}\right)\left|f_{1}^{\prime} \cdots \hat{f_{j}^{\prime}} \cdots f_{n}^{\prime}\right|^{2} .
$$


For a simple mapping

$$
w^{j}=z^{j}
$$

we have

$$
\Omega=\frac{r^{2 n-1}}{\left(1+r^{2}\right)^{n+1}} d r \wedge \Theta, \quad B=\frac{n+r^{2}}{\left(1+r^{2}\right)^{n}} . \quad(|z|=r)
$$

Hence

$$
\begin{gathered}
v\left(D_{r}\right)=\int_{D_{r}} \Omega=\frac{1}{2 n} I_{2 n-1}\left(\frac{r^{2}}{r^{2}+1}\right)^{n} . \\
T_{1}(r)=\int_{r_{0}}^{r} \frac{v\left(D_{r}\right)}{r} d r=\frac{1}{4 n} I_{2 n-1}\left[\log \left(r^{2}+1\right)+\right. \\
\left.\quad+\sum_{k=1}^{n-1}(-1)^{k-1} \frac{1}{k}\left(\begin{array}{c}
n-1 \\
k
\end{array}\right) \frac{1}{\left(r^{2}+1\right)^{k}}\right]_{r_{0}}^{r} \\
Y(r)=\frac{1}{I_{2 n-1}} \int_{s_{r}} B r^{2 n-2} \Theta=\frac{r^{2 n-2}\left(n+r^{2}\right)}{\left(1+r^{2}\right)^{n}} .
\end{gathered}
$$

As $r \rightarrow \infty, T_{1}(r)=O(\log r), Y(r) \rightarrow 1$ and (5.3) is an example of theorem B. On the other hand we have for $n>1$

$$
\begin{aligned}
& T_{2 n-1}(r)=\frac{I_{2 n-1}}{2 n} \int_{r_{0}}^{r} \frac{r d r}{\left(1+r^{2}\right)^{n}}=\left[-\frac{I_{2 n-1}}{4 n(n-1)} \cdot \frac{1}{\left(1+r^{2}\right)^{n-1}}\right]_{r_{0}}^{r} \\
& U(r)=\int_{D\left(r_{0}, r\right)} \frac{B}{r} d r \cdot \Theta=I_{2 n-1} \int_{r_{0}}^{r} \frac{n+r^{2}}{r\left(1+r^{2}\right)^{n}} d r .
\end{aligned}
$$

Hence $T_{2 n-1}(r)$ and $U(r)$ is finite as $r \rightarrow \infty$ and (5.3) is not an example of theorem A.

In theorem $\mathrm{A}$ the case $k=2 n-1$ is treated and the result can be got by omitting the terms

$$
-\int_{B_{r}} v \sin ^{-2 n+2} t \cdot \Delta \bar{\Delta} \Theta, \quad-\int_{D\left(r_{0}, r\right)} 2 n v r^{-1} \sin ^{-2 n+2} t \cdot \Delta \bar{\Delta} d r \Theta
$$

in $J_{2 n-1}$. For the simple mapping (5.3) these terms have the same order with the remaining terms, while we have omitted the term containing $C_{0}-\frac{1}{2}(k+1)$ $\overline{\Delta \Delta}=C_{0}-\bar{\Delta}$ in theorem $\mathrm{B}$, which is natural for the mapping (5.3).

\section{References}

[1] S. S. Chern, The integrated form of the first main theorem for complex analytic mappings in several complex variables, Ann. of Math., 71 (1960), 536-551.

[2] S. S. Chern, Holomorphic mapping of complex manifolds, Enseignement Math., 7 (1961), 552-560. 
[3] M. Kurita, On normal contact metric manifolds, J. Math. Soc. Japan, 15 (1963), 304-318.

[4] S. Sasaki and Y. Hatakeyama, On differentiable manifolds with contact metric structures, J. Math. Soc. Japan, 14 (1962), 249-271.

Mathematical Institute

Nagoya University 